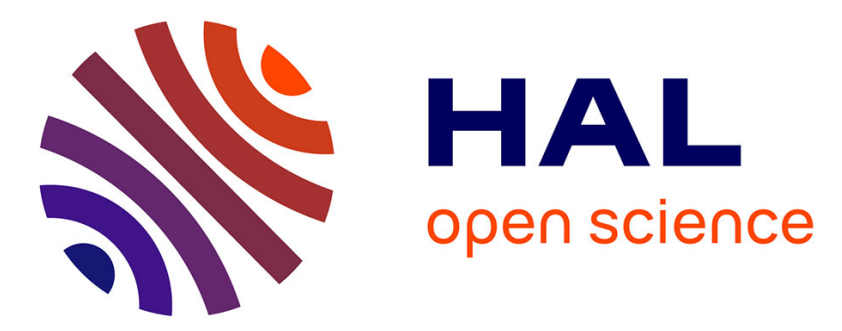

\title{
Understanding conductivity in molecular switches: a real space approach in octaphyrins
}

\author{
T. Woller, N. Ramos-Berdullas, M. Mandado, M. Alonso, F. de Proft, J.
}

Contreras-García

\section{- To cite this version:}

T. Woller, N. Ramos-Berdullas, M. Mandado, M. Alonso, F. de Proft, et al.. Understanding conductivity in molecular switches: a real space approach in octaphyrins. Physical Chemistry Chemical Physics, 2016, 10.1039/C5CP07411H . hal-01274654

\section{HAL Id: hal-01274654 https://hal.sorbonne-universite.fr/hal-01274654}

Submitted on 16 Feb 2016

HAL is a multi-disciplinary open access archive for the deposit and dissemination of scientific research documents, whether they are published or not. The documents may come from teaching and research institutions in France or abroad, or from public or private research centers.
L'archive ouverte pluridisciplinaire HAL, est destinée au dépôt et à la diffusion de documents scientifiques de niveau recherche, publiés ou non, émanant des établissements d'enseignement et de recherche français ou étrangers, des laboratoires publics ou privés. 


\title{
Journal Name
}

\section{Understanding conductivity in molecular switches: a real space approach in octaphyrins}

\begin{abstract}
T. Woller, ${ }^{a, b}$ N. Ramos-Berdullas, ${ }^{c}$ M. Mandado, ${ }^{c}$ M. Alonso, ${ }^{a}$ F. de Proft, ${ }^{a}$ and J. Contreras-García ${ }^{b^{*}}$
In recent years, expanded porphyrins have emerged as a promising class of $\pi$-conjugated molecules that display unique electronic, optical and conformational properties. Several expanded porphyrins can switch between planar and twisted conformations, which have different photophysical properties. Such a change of topology involves a Hückel-Möbius aromaticity switch in a single molecule and it can be induced by solvent, $\mathrm{pH}$ and metallation. These features make expanded porphyrins suitable for the development of a novel type of molecular switches for molecular electronic devices. Octaphyrins consisting of eight pyrrole rings, exhibit twisted-Hückel, Möbius and Hückel $\pi$-conjugation topologies depending on the oxidation and protonation state, with distinct electronic structures and aromaticity. Our working hypothesis is that a significant change in the conductance of expanded porphyrins will be observed after the topology switching. Despite the potential of Hückel-Möbius systems as conductance switches, the relationship between the conductance and the molecular topology is not yet understood. We have explored the performance of local descriptors of conductivity in simple molecules, as well as the relationship with conductance. Since these indexes provide a qualitiative measure of delocalization and conductance in the probe molecules, we have carried out a local analysis of electrical conductance changes as a function of the $\pi$-conjugation in two examples. In one of them, the locality of the electronic changes ensures the ability of these indexes to describe the conductance as local. Moreover, it enables to identify which conformational switch would be more efficient from an electronic device perspective. However, we also show that it is not always possible to reduce conductance changes to one bond, and in those molecules where a deep rearrangement occurs far from the structural perturbation, local measures show a limited efficiency. This is a first step for the description of the connection between the molecular structure and conductance in molecular switches.
\end{abstract}

\section{Introduction}

Nowadays, research in molecular electronics focuses on the inclusion of small active molecules into electronic circuits. In those systems, the molecular switching elements carry out electronic functions such as diodes, transistors and switches. A spectacular example is a molecular four-level conductance switch based on single proton transfer in a porphyrin derivative. ${ }^{1}$ In molecular electronics, the investigation of molecular switching elements with a panel of functions provides future prospects for miniaturization in the field of nanotechnology. Molecular switches are regarded as the most basic component in molecular electronic devices that can reverse from an active/on state to a passive/off state. The switch between two or more states with distinct properties is

\footnotetext{
a. Eenheid Algemene Chemie (ALGC), Vrije Universiteit Brussel (VUB). Pleinlaan 2, 1050 Brussels (Belgium)

${ }^{b .}$ Laboratoire de Chimie Théorique. 4,PI. Jussieu. 75252 Paris cedex 05

c. Department of Physical Chemistry, University of Vigo, Lagoas-Marcosende $s / n$, 36310 Vigo, Spain
}

Electronic Supplementary Information (ESI) available: [details of any supplementary information available should be included here]. See DOI: $10.1039 / x 0 x x 00000 x$ triggered by external stimuli such as light, $\mathrm{pH}$ or voltage. ${ }^{2}$ As opposed to normal switches, molecular switches are extremely tiny and their application in nanotechnology, biomedicine and computer chip design opens up a new horizon.

In recent years, there has been a considerable growth in research on expanded porphyrins., ${ }^{3,4}$ Owing to their tunable photophysical and chemical properties with external stimuli, expanded porphyrins represent a very promising platform to develop molecular switches for molecular electronic devices. ${ }^{5-7}$ The most fascinating property of expanded porphyrins is their capacity to switch between several distinct $\pi$-conjugation topologies with different absorption and emission spectra. ${ }^{8}$ Interestingly, recent experimental studies ${ }^{9}$ proved that the photophysical properties of porphyrins are closely related to their molecular conformation and macrocyclic aromaticity. Such a change of topology is coupled to a Hückel-Möbius aromaticity switch in a single molecule. Moreover, the topology switching is achieved by variation of one internal dihedral angle and, if properly controlled, can provide access to molecular switches with unique optical and magnetic properties. However, despite their potential, the design of molecular switches based on expanded porphyrins has only been scarcely investigated. ${ }^{6,10,11}$ 
Octaphyrins, which are composed of eight pyrrole rings, hold a unique place among expanded porphyrins: their figure-eight conformation displays two-metal coordination and intrinsic chirality. ${ }^{8,12,13}$ The aim of this paper is to obtain qualitative insight into the conductance of octaphyrins as a function of their molecular conformation using the concept of bond metallicity. More specifically, understanding the locality of conductance changes with respect to the variation of the internal dihedral angles is crucial for the design of improved molecular switches.

However, the most common measures of metallicity are not local. For example, a metallic bond will be associated with a large local density of states at the Fermi level. Similarly, it is known that the "nearsightedness" of the off-diagonal terms in the first-order density matrix increases as the band gap increases. ${ }^{14-17}$ The problem with these forms is that they require non-local information and this means that the connexion with real space (molecular structure, bonds and dihedral angles) is lost. Several local indicators have been recently proposed in the literature. ${ }^{18-20}$ This contribution aims at understanding and establishing the structure-property relationship between molecular conformation and molecular conductance in octaphyrins using the concept of bond metallicity, which so far has only been applied to solids.

Our paper will proceed as follows. We will start by introducing the various indexes related to bond metallicity developed in the literature. Then, we will perform a proof of principle. We will test the implementation of these indexes on different molecular systems in order to assess their performance and limitations, their ability to reveal conductance and the subtleties of a topological switch. Finally, these indices will be applied to Hückel and Möbius conformations of octaphyrins in order to understand the locality of the conductivity changes upon dihedral rotation and when this assumption stays valid. This insight might help in the design of improved topological switches.

\section{Methodology}

There have been several approaches to tackle conductivity from local measures. The most common approach is to resort to density-related values at the bond critical point as originated from Atoms In Molecules (AIM). ${ }^{21-26}$

Within AIM, one commonly characterizes chemical bonds using the properties of the electron density at the bond critical point $(b c p)$. In an ordinary molecule, the electron density has maxima (cusps) at the nuclei and decays exponentially as the electron density moves away from the nuclei. The resulting topology looks like an assemblage of mountains, each of which is identified as an atom. The mountain pass would then represent the bond, and its lowest point identifies the presence of such a connection, which is where their name (bond critical point, bcp) stems from.

A somewhat different approach, which requires comparing different critical points, was proposed by Mori-Sanchez et al. ${ }^{27}$ Although this approach allows one to distinguish covalent, ionic, and metallic bonds, it does not provide a truly local measure of metallicity, as related to a given bond. Thus, we will concentrate on the measures uniquely related to bcps.

In 2009, Jenkins proposed a bond metallic index based on the electron density and its Laplacian at the bond critical point $\rho\left(r_{b c p}\right)$ and, $\nabla^{2} \rho\left(r_{b c p}\right)$, respectively. The sign of the laplacian determines the interaction type, distinguishing from closed shell, such as ionic or metallic (positive laplacian) and shared or covalent interactions (negative laplacian). Thus when looking at these measures and metallicity, one concentrates only on bcps with positive laplacian values. ${ }^{28}$

$$
\xi_{j}\left(r_{b c p}\right)=\frac{\rho\left(r_{b c p}\right)}{\nabla^{2} \rho\left(r_{b c p}\right)}
$$

However $\xi_{\mathrm{j}}$ is neither dimensionless nor defined for singular Laplacian. Thus a revised version overcoming these flaws was proposed: ${ }^{19}$

$$
\xi_{m}\left(r_{b c p}\right)=\frac{36\left(3 \pi^{2}\right)^{2 / 3} \rho\left(r_{b c p}\right)^{2 / 3} \xi_{j}\left(r_{b c p}\right)}{5}
$$

Based on the value of $\xi_{\mathrm{m}}$ and $\xi_{\mathrm{j}}$, at a $b c p$ with positive laplacian (i.e. at non covalent interactions), metallic bonds can be distinguished from non-metallic bonds. For instance, metallic bonds are characterized by $\xi_{\mathrm{m}}$ superior to 25 whereas $\xi_{\mathrm{m}}$ values between 5 and 25 indicate a partial metallic character. Weak metallic bonds are identified by $\xi_{\mathrm{m}}$ values between 1 and 5 . When $\xi_{\mathrm{m}}$ is inferior to 1 , the bonds present a nonmetallic character.

Still focusing on the bcps, local metallicity measures have also been introduced in terms of kinetic energy densities and delocalization measures. In our work, we will consider three types: the Electron Localization Function or ELF, kinetic energy density based measures (where ELF could be included according to Savin's interpretation ${ }^{29}$ ) and the local covariance.

According to the formulation of Becke and Edgecombe, ${ }^{30} \mathrm{ELF}$ measures the probability of finding an electron in the vicinity of another electron with the same spin.:

$$
E L F=\frac{1}{1+\left(\frac{D}{D_{h}}\right)^{2}}=\frac{1}{1+\chi^{2}}
$$

In Savin's interpretation ${ }^{29}$, D corresponds to the difference between the true kinetic energy and Weizsäcker's kinetic energy (i.e. for a bosonic system), whereas $D_{h}$ denotes the Thomas-Fermi kinetic energy. The variable $\chi$ provides a measure of the effect of the Pauli principle in the kinetic energy density.

In highly localized regions such as covalent bonds or nuclei, ELF approaches 1 . ELF is equal to 0.5 in the uniform electron gas (i.e. in a perfectly delocalized system). ELF can be used to 
understand electron delocalization when analysed in between ELF basins ${ }^{31}$ (e.g. around intermolecular bcps). In these cases, high ELF values identify delocalization in between localized regions. ${ }^{32}$ This explains why $\xi_{\mathrm{m}}$ is positively correlated with the electron localization function at positive laplacian bcps. ${ }^{33}$

In addition, localized orbitals can also be a useful tool for metallicity measures. ${ }^{34}$ The Localized Orbital Locator (LOL or $t_{s b}$ ) reflects the localization of orbitals in electronic systems compared with the uniform electron gas and presents values superior to 1 for highly localized systems such as covalently bound valence electrons. ${ }^{35-36}$

$$
t_{s b}\left(r_{b c p}\right)=\frac{2 \tau_{T F}\left(r_{b c p}\right)}{3 \tau\left(r_{b c p}\right) \rho\left(r_{b c p}\right)}
$$

Where $\tau$ refers to the kinetic energy density and $\tau_{T F}$ to the kinetic energy density of the homogeneous electron gas (Thomas-Fermi). Values of $t_{s b}<1$ imply that the region is depleted in electrons with respect to the uniform gas. ${ }^{37}$ Therefore, as the bond metallicity and $t_{s b}$ are positively correlated to the electron density at the bond critical point, they will decrease with increasing delocalization.

In addition, the delocalization indicator, present in the definition of ELF, can also be rewritten in terms of the electron density and the local temperature. The local temperature corresponds to the ratio of kinetic energy density over the electron density and is thus inversely proportional to the bond metallicity. $^{38}$

$$
\chi\left(r_{b c p}\right)=\frac{\tau(R)-\tau_{w}(R)}{\tau_{T F}}=\frac{\frac{3}{2} \rho(R) \theta(R)-\tau_{w}(R)}{\tau_{T F}}
$$

As demonstrated by S. Jenkins and co-workers, it is possible to apply the gradient expansion approximation to explore the link between the different metallicity measures. In the gradient expansion approximation, the kinetic energy density is expanded as the sum of the Thomas-Fermi ${ }^{39-40}$ and Weizsäcker's kinetic energy densities ${ }^{40}$ and the Laplacian. ${ }^{41}$

$$
\begin{gathered}
\tau=\tau_{T F}+\frac{1}{9} \tau_{W}-\frac{1}{6} \nabla^{2} \rho \\
\tau_{T F}=\frac{3\left(3 \pi^{2}\right)^{2 / 3} \rho\left(r_{b c p}\right)^{5 / 3}}{10} \\
\tau_{W}=\frac{\nabla \rho \nabla \rho}{8 \rho}
\end{gathered}
$$

As a result, we can approximate the localized orbital locator $t_{\mathrm{sb}}$ in terms of the bond metallicity index:

$$
t_{s b} \approx \frac{\xi_{m}\left(r_{b c p}\right)}{1+4 \xi_{m}\left(r_{b c p}\right)}
$$

Since the electron delocalization function in ELF can be expressed in terms of the local temperature, it is also possible to establish a link between ELF and the bond metallicity. At bond critical points, we observe that the electron delocalization is related to the inverse of the bond metallicity.

$$
\chi\left(r_{b c p}\right) \approx 1+\frac{4}{\xi_{m}\left(r_{b c p}\right)}
$$

One has to keep in mind that the gradient expansion approximation is only valid for regions with a low electron localization $\left(t_{\mathrm{sb}}<1\right)$ and does not provide reliable results for highly localized systems ( $\left.t_{\mathrm{sb}}>>1\right)$. Thus, as long as we stay in weak interactions (positive laplacian, low density), the approximation should hold.

\section{Computational details}

All calculations were performed with the Gaussian suite of programs. ${ }^{42-43}$ The geometries of the different conformations of unsubstituted [36]octaphyrins were optimized and characterized by harmonic frequency calculations with the M06 functional and the $6-31 G(d, p)$ basis set. Single point calculations with the extended $6-311+G(d, p)$ basis set were also performed in order to compute more accurate electronic energies. One-dimensional relaxed scans were computed at the M06/6-31G(d,p) level of theory in order to locate the transition states and interconversion pathways for the most stable conformations. Metallicity measures were implemented in Topmod. ${ }^{44}$ An in-house code was used for the search of critical points.

\section{Results}

The optical and aromatic properties of expanded porphyrins are highly dependent on their $\pi$-conjugation topology. 12,45 Since aromaticity is associated with electron delocalization, ${ }^{46}$ our ultimate aim is to check how the conductivity changes upon the topology switching. However, since the approaches introduced above have been mainly applied to solids, ${ }^{18-20,33}$ the first section is devoted to a proof of principle of the applicability of the local metallicity indexes to small molecules. Based on the results from the first section, we then analyse how these indices change as a function of the topology in octaphyrins and check the locality of the changes introduced by molecular conformation.

\section{Proof of principle}

This proof of principle addresses three fundamental questions whose answers determine the applicability of the local concept of metallicity to octaphyrins: (i) check which indices correctly reflect local metallicity (and their mutual coherency), specially upon molecular twisting (ii) check whether they reflect conductivity changes in a qualitative manner and (iii) evidence the locality of conductivity in small molecular switches and thus, the applicability of the indexes to octaphyrins.

\section{(i) Comparison of indexes}


To check the performance of local metallicity indexes in molecules, we have chosen a set of small organic dimers. Our first test set is a series of complexes (Figure 1) consisting of hexafluorophenyl and a small molecule $\left(\mathrm{LiH}, \mathrm{FH}, \mathrm{NH}_{3}, \mathrm{H}_{2} \mathrm{O}\right.$, $\mathrm{HCN}, \mathrm{CNH}$ ) because of the wide range of interactions as well as their simplicity. ${ }^{47}$ Optimizations were done with the CAMB3LYP/6-31G(d,p) functional to avoid delocalization errors in the complexes. We have tested three types of indexes introduced in the Methodology section as well as the performance of their approximate gradient expansion expressions.

The aim of this test is to check the delocalization within the system and the ability of the above indexes to reflect the delocalization through space, capabilities that are necessary to analyse cyclic systems.

According to Table 1 , the $\mathrm{LiH}$ - and the $\mathrm{FH}$-hexafluorophenyl complexes present the highest and the lowest $\xi_{\mathrm{m}}$ and ELF values, respectively. According to their $\xi_{\mathrm{m}}$ values, none of the system presents a metallic bond but those with higher $\xi_{\mathrm{m}}$ values tend to display non-covalent bonds which would fall in

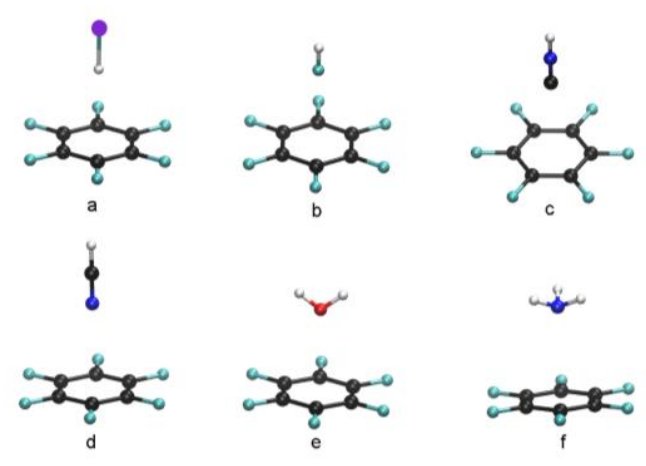

Figure 1. Hexafluorophenyl complexes. In top-bottom, left-right order: $\mathrm{LiH}, \mathrm{FH}, \mathrm{CNH}, \mathrm{HCN}, \mathrm{H}_{2} \mathrm{O}$ and $\mathrm{NH}_{3}$

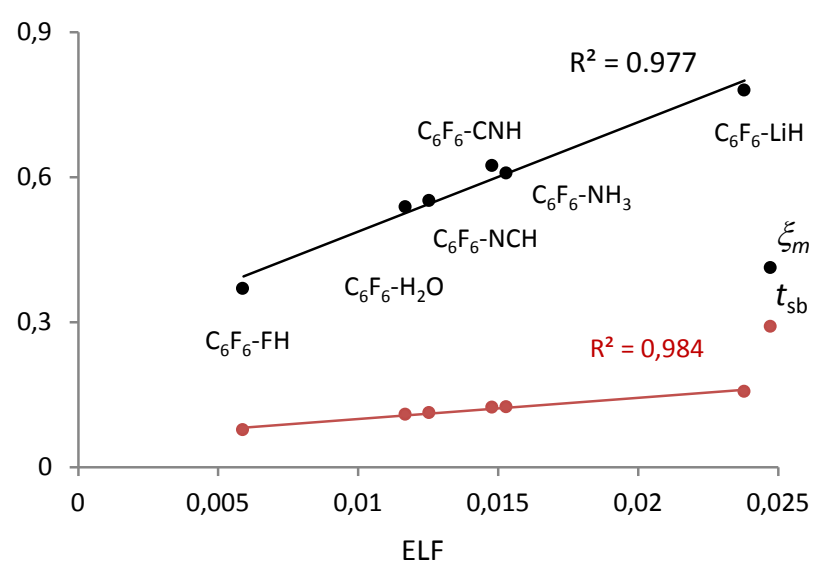

Figure 2. Correlation between bond metallicity and the localized orbital locator with ELF values for systems in Figure 1. the very weak metallic character range. The trend can be understood in terms of the electronegativity of the atom and the delocalized nature of the electrons facing the ring. In the case of $\mathrm{LiH}$, the $\mathrm{H}$ atom faces the ring, where it is the atom $\mathrm{X}$ in the $\mathrm{X}(\mathrm{Y}) \mathrm{H}$ complexes the one that is closer to the ring in the rest of the cases. $\mathrm{H}$ atom, being very diffuse, comes first in the series. Then the rest of the complexes are ordered in terms of electronegativity: $\mathrm{C}, \mathrm{N}, \mathrm{O}$ and $\mathrm{F}$. It is interesting to note that $\mathrm{NH}_{3}$ and $\mathrm{CNH}$ lay very close in all measures.

As shown in Figure 2, the measures of metallicity based on the kinetic energy $t_{s b}$ and the bond metallicity $\xi_{m}$ present an excellent correlation with ELF values at the bond critical point. According to Table 1 and Figure 2, the orbital localization and the bond metallicity increase with the ELF values from the $\mathrm{C}_{6} \mathrm{~F}_{6}-\mathrm{FH}$ to the $\mathrm{C}_{6} \mathrm{~F}_{6}-\mathrm{LiH}$ complex. Approximating this relationship by a straight line, correlations coefficients close to 1 are obtained, much simpler than the relationship expected from the Eqs. 8 and 9.

Table SI.1 collects the metallicity measures computed with the gradient expansion approximation (GEA). In general, the expansion provides results in good agreement with the original data (Table 1). Owing to the excellent correlation between ELF and the measures of metallicity within the GEA framework $\left(R^{2}\left(\xi_{\mathrm{m}}\right)=0.967\right.$ and $\left.R^{2}(\chi)=0.972\right)$, we will employ the GEA approximation of $\xi_{\mathrm{m}}$ and $\chi$ to evaluate the metallicity in the next systems.

Since we want to apply these indexes to analyse the changes of conductivity along the rotation of $\mathrm{C}-\mathrm{C}$ bonds in octaphyrins, it is important to first check whether they are able to go beyond the identification of changes in delocalization among different compounds and characterize relative delocalization upon rotation of a given molecule. We have analysed the twisting in dipyrromethene since it shows a similar structure to octaphyrins. Dipyrromethene presents a bond critical point between its imino and amino group (Figure 3 ) until the central torsional angle reaches $50^{\circ}$, value at which the hydrogen bond vanishes. This is an intrinsic drawback of these local quantities: they cannot be measured beyond $b c p$ coalescence. ${ }^{48}$

Table 1.Metallicity measures of CAM-B3LYP/6-31G(d,p) optimized structures at the bond critical points between the small molecule and the aromatic ring (systems in Figure 1).

\begin{tabular}{c|l|c|c|c|}
\cline { 2 - 5 } & Name & $\xi_{m}$ & ELF & $t_{\text {sb }}$ \\
\hline a & $\mathrm{C}_{6} \mathrm{~F}_{6}-\mathrm{LiH}$ & 0.779 & 0.024 & 0.156 \\
$\mathrm{c}$ & $\mathrm{C}_{6} \mathrm{~F}_{6}-\mathrm{CNH}$ & 0.608 & 0.015 & 0.125 \\
$\mathrm{f}$ & $\mathrm{C}_{6} \mathrm{~F}_{6}-\mathrm{NH}_{3}$ & 0.623 & 0.015 & 0.123 \\
$\mathrm{~d}$ & $\mathrm{C}_{6} \mathrm{~F}_{6}-\mathrm{NCH}$ & 0.551 & 0.012 & 0.113 \\
$\mathrm{e}$ & $\mathrm{C}_{6} \mathrm{~F}_{6}-\mathrm{H}_{2} \mathrm{O}$ & 0.538 & 0.012 & 0.109 \\
$\mathrm{~b}$ & $\mathrm{C}_{6} \mathrm{~F}_{6}-\mathrm{FH}$ & 0.369 & 0.006 & 0.077 \\
\hline
\end{tabular}




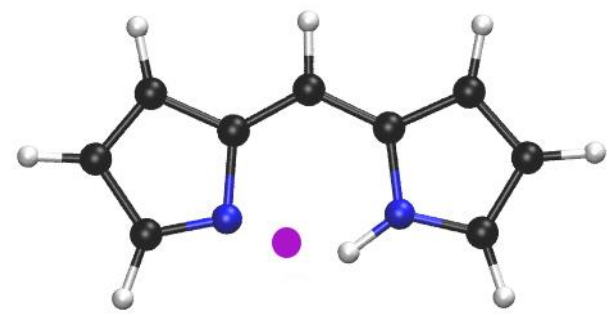

Figure 3. Bond critical point representing the hydrogen bonding in the dipyrromethene molecule.

Table 2. Measures of metallicity for dipyrromethene with different dihedral angles.

\begin{tabular}{c|cccc}
\hline $\begin{array}{c}\text { Dihedral } \\
\text { angle }\end{array}$ & ELF & $\xi_{\mathrm{m}}$ & $\mathrm{t}_{\mathrm{sb}}$ & $\mathrm{d}^{\mathrm{a}}$ \\
\hline 0 & 0.122 & 2.257 & 0.362 & 2.017 \\
10 & 0.119 & 2.215 & 0.356 & 2.031 \\
20 & 0.108 & 2.082 & 0.342 & 2.069 \\
30 & 0.090 & 1.854 & 0.317 & 2.139 \\
40 & 0.067 & 1.519 & 0.275 & 2.256 \\
\hline
\end{tabular}

The measures of metallicity of the dipyrromethene indicate that the metallicity decreases upon rotation. This can be understood as a reduction of $\pi$-conjugation, due to an elongation of the hydrogen bond (Table 2). Towards the end of the rotation, there is an important change in slope of all the metallicity indexes (Figure 4). This is due to the fact that the hydrogen bond $b c p$ is on the verge of disappearing.

\section{(ii) Delocalization and conductivity}

Once the ability of these indexes to identify delocalization has been settled, the question of whether this can be related to conductivity remains open. Some of the present authors, have recently investigated the electronic properties of $p$-xylylene (PX1 and PX2) and $p$-phenylene (PP) chains at different finite bias voltage (Figure 5). ${ }^{49}$ Due to the covalent bond between

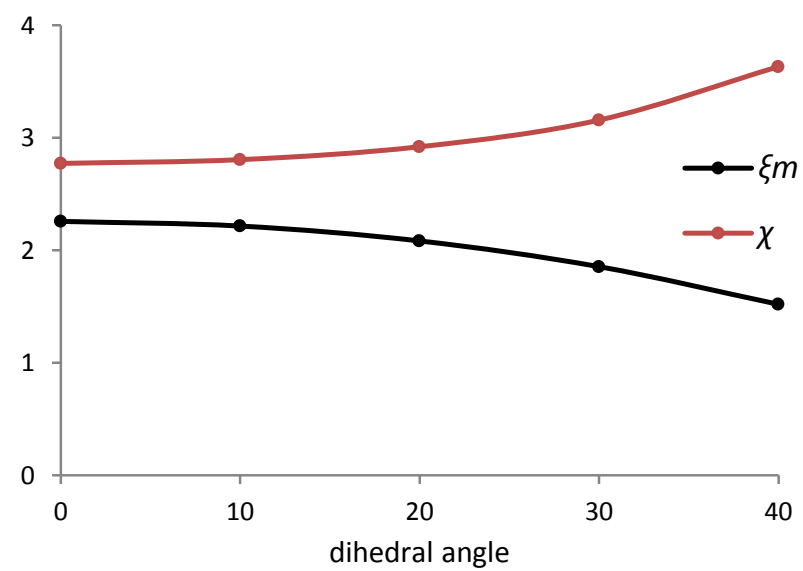

Figure 4.Evolution of several indices of metallicity with the dihedral angle of dipyrromethene.

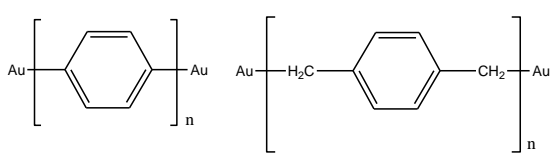

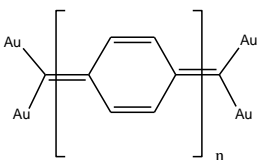

PX2
Figure 5.Investigated $p$-phenylene (PP) and $p$-xylylene (PX1 and PX2) units.

the gold atom and the carbon chain in PX, xylylene presents higher conductivity than PP, where the bond to the benzene unit is direct. Moreover, the response to an applied external voltage is different for these three structures. For instance, the applied external voltage induces a higher delocalization in PX2 as the voltage and the number of ring increases. Instead, the external voltage destroys the delocalization in PX1 and PP, which leads to a reduction of the delocalization as the number of rings and the voltage increase.

We have compared the metallicity indexes with the intensity and voltage results from Ref. 49. All the measures of metallicity were computed at the junction between the gold atom and the organic part of the molecule. The bond metallicity, the delocalization indicator computed with the gradient expansion approximation, the voltage and the current intensity are collected in Table 3 for $p$-phenylene with one and two subunits. Owing to the presence of $\mathrm{Au}$ atoms, the bond between $\mathrm{Au}$ and the organic moiety lies in the intermediary metallic range $\left(\xi_{m}\right.$ within 5-25). The metallicity of the system increases with the current intensity (Figure 6) whereas the delocalization of electrons is inversely proportional to the current intensity (Figure SI.3). Both measures of metallicity ( $\xi_{m}$ and $\chi)$ are correlated to the current intensity $\left(R^{2}>0.98\right)$ and at a lower extent to the voltage $\left(R^{2}>0.90\right)$ (see Figure SI.4). Moreover, the phenylene chain with two ring subunits displays higher metallicity than its homologous chain with one subunit (Figure 6). In addition, the delocalization of the electrons decreases with the applied voltage, more rapidly in $p$ phenylene with one subunit, falling very rapidly at high voltage (Figure SI.5b). This reduction of the delocalization indicator is in agreement with the results from Ref. 49, which showed that the delocalization was destroyed at higher voltage in PP. ${ }^{49}$

Table 3. Induced current density, metallicity index and delocalization indicator for $p$-phenylene (PP) with one (n1) and two (n2) phenyl rings. Intensity in microampers and voltage in volts.

\begin{tabular}{l|llllll}
\hline $\mathrm{V}$ & $\mathrm{I}(\mathrm{n} 1)$ & $\xi_{\mathrm{m}}(\mathrm{n} 1)$ & $\chi(\mathrm{n} 1)$ & $\mathrm{I}(\mathrm{n} 2)$ & $\xi_{\mathrm{m}}(\mathrm{n} 2)$ & $\chi(\mathrm{n} 2)$ \\
\hline 0 & 0.000 & 15.545 & 1.257 & 0.000 & 15.672 & 1.255 \\
0.5 & 0.072 & 12.570 & 1.318 & 0.033 & 15.702 & 1.255 \\
1.5 & 2.093 & 13.540 & 1.295 & 0.814 & 16.056 & 1.249 \\
2.5 & 9.766 & 14.812 & 1.270 & 3.891 & 16.833 & 1.238 \\
3.5 & 27.398 & 22.000 & 1.182 & 10.317 & 18.180 & 1.220 \\
\hline
\end{tabular}




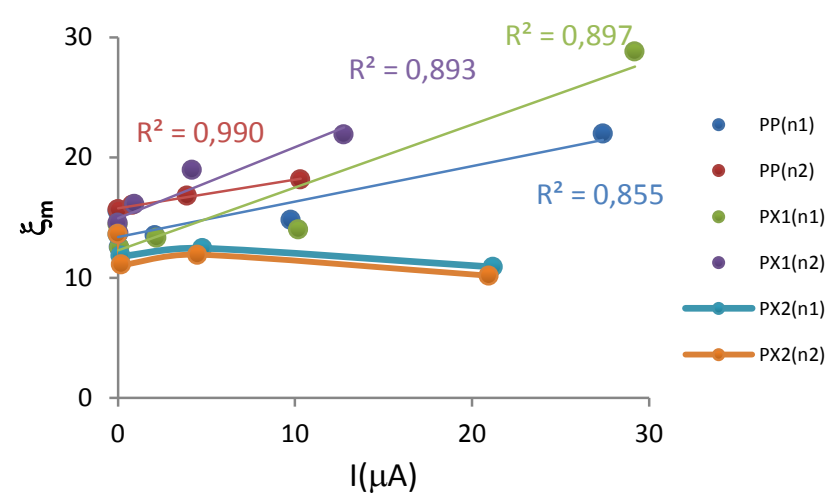

Figure 6.Relation of the bond metallicity with the induced current for $\mathrm{PP}, \mathrm{PX} 1$ and PX2.

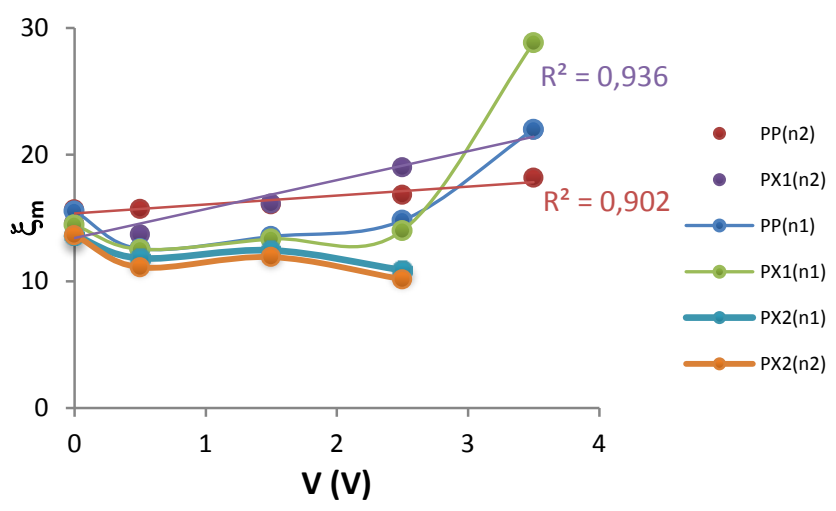

Figure 7. Relation of the bond metallicity with the applied voltage for $\mathrm{PP}, \mathrm{PX} 1$ and PX2.

$p$-Xylylene PX1 behaves similarly with the external perturbation as PP because the bond metallicity increases with induced current and applied voltage (Figures 6 and SI.6). However, PX1 possesses higher molecular conductance, which can be related to its higher metallicity (Table 4). Although our index faces some troubles to estimate the metallicity measures at $\mathrm{V}=0.5 \mathrm{~V}$, it provides a qualitative picture of the evolution of the system which is in agreement with the observations of Ramos-Berdullas et $a .^{49}$

PX1 follows a similar trend as PP with the voltage. The metallicity index is inversely related to the voltage and the

Table 4. Induced current density, metallicity index and delocalization indicator for $p$-xylylene (PX1) with one (n1) and two (n2) phenyl rings. Intensity in microampers and voltage in volts.

\begin{tabular}{l|llllll}
\hline $\mathrm{V}$ & $\mathrm{I}(\mathrm{n} 1)$ & $\xi_{\mathrm{m}}(\mathrm{n} 1)$ & $\chi(\mathrm{n} 1)$ & $\mathrm{I}(\mathrm{n} 2)$ & $\xi_{\mathrm{m}}(\mathrm{n} 2)$ & $\chi(\mathrm{n} 2)$ \\
\hline 0 & 0.000 & 14.451 & 1.277 & 0.000 & 14.594 & 1.274 \\
0.5 & 0.074 & 12.530 & 1.319 & 0.030 & 13.700 & 1.292 \\
1.5 & 2.190 & 13.342 & 1.299 & 0.921 & 16.123 & 1.248 \\
2.5 & 10.198 & 14.014 & 1.285 & 4.186 & 18.989 & 1.231 \\
3.5 & 29.202 & 28.823 & 1.139 & 12.758 & 21.941 & 1.182 \\
\hline
\end{tabular}

number of subunits (Figures 6 and SI.6). However, the localization of electrons is more pronounced in PX1 than in PP (Figure 7) because the gold atoms are covalently bound to methyl groups, which seem to be more affected by the external voltage. Nevertheless, both PP and PX1 behave as bulk semiconductors.

Unlike PP and PX1, the bond metallicity index of PX2 decreases with increasing applied voltage (Figure SI.7) while the induced current still increases with the voltage (Table 5). However, PX2-n2 presents lower current intensity than PX2-n1, coupled in general to higher metallicity values (Figure 6). In comparison with PP and PX1, PX2 presents a lower bond metallicity index at the junction between gold and the $\mathrm{sp}^{2}$ carbon atom because the electrons are more delocalized in the whole structure. The evaluation of the bond metallicity is more problematic for the PX2-n2 structure at $0.5 \mathrm{~V}$ because there is a drop in bond metallicity and an increase in electron delocalization (Figures 7 and SI.7). Nevertheless, the bond metallicity still provides the correct qualitative picture because it is able to grasp the different behaviour of PX2 with respect to PP and PX1.

In contrast to PX1 and PP, the delocalization index $\chi$ generally increases with the voltage and the induced current intensity in PX2 (Figures 7 and SI.7). This opposite behaviour of the delocalization of the electrons indicates that higher voltage enhances the electrons delocalization in PX2. Moreover, the inclusion of the additional subunits in PX2 also promotes the electron delocalization in the whole molecule. RamosBerdullas et al. reported that the molecular response of PX2 to the applied voltage was not similar to that of a bulk semiconductor. $^{49}$

Therefore, we can say that the bond metallicity indexes provide a qualitative picture of the molecular conductance and the electronic properties of molecular semi-conductors. However, further analysis is needed in order to predict the response of a molecule belongs in the presence of metallic junctions, e.g. whether delocalization increases or decreases with voltage.

Table 5. Induced current density, metallicity index and delocalization indicator for $p$-xylylene (PX2) with one (n1) and two (n2) phenyl rings. Intensity in microampers and voltage in volts. Results at $\mathrm{V}=3.5 \mathrm{~V}$ have not been included due to problems with the critical point search.

\begin{tabular}{l|llllll}
\hline $\mathrm{V}$ & $\mathrm{I}(\mathrm{n} 1)$ & $\xi_{\mathrm{m}}(\mathrm{n} 1)$ & $\chi(\mathrm{n} 1)$ & $\mathrm{I}(\mathrm{n} 2)$ & $\xi_{\mathrm{m}}(\mathrm{n} 2)$ & $\chi(\mathrm{n} 2)$ \\
\hline 0 & 0.000 & 13.617 & 1.294 & 0.000 & 13.646 & 1.293 \\
0.5 & 0.162 & 11.829 & 1.338 & 0.172 & 11.112 & 1.359 \\
1.5 & 4.761 & 12.450 & 1.321 & 4.492 & 11.917 & 1.335 \\
2.5 & 21.196 & 10.888 & 1.368 & 20.948 & 10.168 & 1.393 \\
\hline
\end{tabular}




\section{(iii) Is delocalization local in octaphyrins?}

Finally, we have assessed whether the evolution of the bond metallicity is local in macromolecular systems such as porphyrins. This last part of the proof of principle aims at exploring the local character of the metallicity measures on a Hückel-Möbius transition in regular porphyrin. According to the value of the dihedral angle $d$, porphyrin presents a Hückel $\left(d<90^{\circ}\right)$ or a Möbius topology $\left(d>90^{\circ}\right)$. The Hückel topology contains formal cis bonds in the smallest macrocyclic pathway, whereas in the Möbius conformation there is one trans bond. The ELF topological analysis reveals that four bond critical points with positive laplacian are present, which correspond to the four hydrogen bonds (see Figure 8).

In Figure 8, the bond critical point 1 (in purple) is always used to refer to the bond critical point that is the closest to the perturbation. If conductivity remains local in these switches, we expect the variation of the measure of the metallicity to be maximal at the perturbation (bcp1) and to become less important with increasing distance from the twist.

We observe that ELF and $\xi_{\mathrm{m}}$ evolve similarly with the dihedral angle (Figures 9 and SI.8). The bond metallicity decreases at all bond critical points except for the bond critical point 2 where the reverse situation is observed. In order to compensate the loss of hydrogen bonds, the hydrogen bond above the bond critical point 2 is strengthened, resulting in increased bond metallicity at this point. The biggest variations in metallicity are observed in $b c p 2$ and $b c p 4$, which are closest ones to the rotating dihedral angle, whereas the metallicity in $b c p 3$ (which is the furthest $b c p$ ) is less affected. It is interesting to note, that the metallicity of $b c p 3$ appears to mimic that of $b c p 1$ before it disappears.

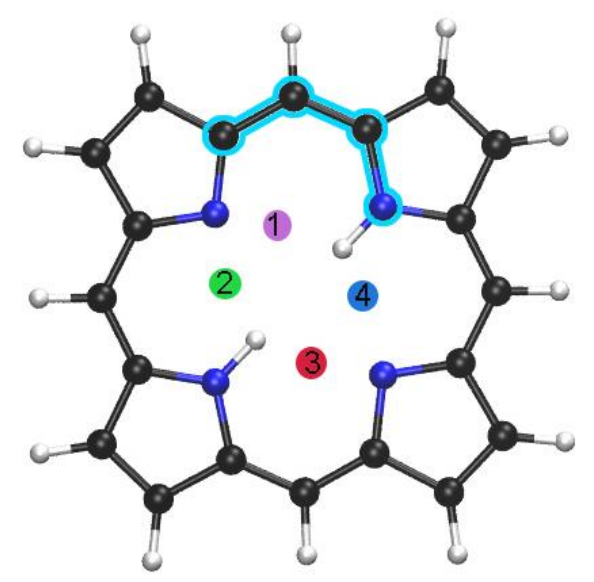

Figure 8. Porphyrin with the investigated bond critical points coloured and numbered according to their position.

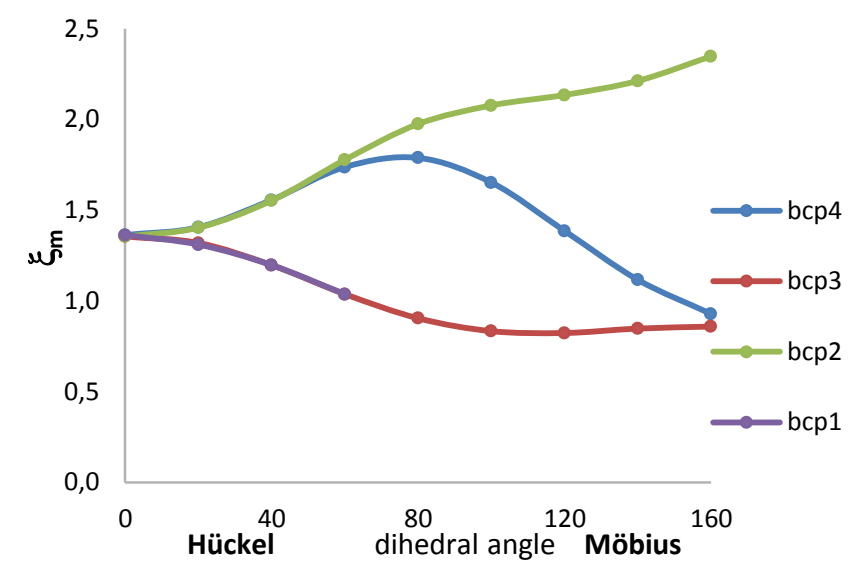

Figure 9. Evolution of the bond metallicity as function of the amplitude of the dihedral angle in porphyrin. Colours follow the pattern in Figure 8 .

\section{Octaphyrins}

In order to gain a qualitative insight into the evolution of the bond metallicity in octaphyrins, two interconversions of octaphyrins were investigated. Since the interconversion between different conformers relies on the variation of one or two dihedral angle(s), ${ }^{50}$ we expect the variation of the bond metallicity to be local, and thus our indices to be applicable. Moreover, based on the study of $p$ phenylene and $p$-xylylene, we have also seen that the bond metallicity and the delocalization indicator are related to the current intensity. Thus, since Möbius and Hückel expanded porphyrins exhibit distinct aromatic and optical properties, the molecular conductance could also be expected to be topologydependent.

Firstly, we investigated a two-step interconversion between two Möbius structures (M1, M2) involving an intermediary Hückel topology $\mathbf{H} \mathbf{1}$ (Figures 10 and 11 ). The rotating dihedral angle is denoted in light blue. In contrast to the previous interconversion, the rotation of the cis dihedral angle in $\mathbf{M} \mathbf{1}$ induces an additional rotation of the neighboring pyrrole ring, giving rise to an extra hydrogen bond in structures $\mathbf{H 1}$ and $\mathbf{M} \mathbf{2}$.

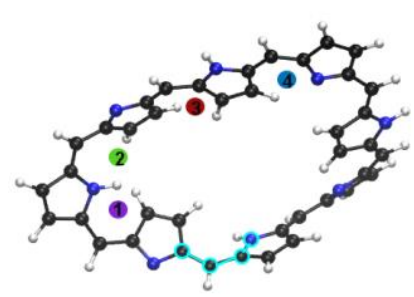

M1

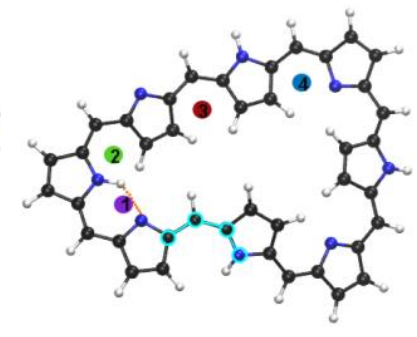

H1
Figure 10.Investigated bond critical points in the molecular switch, where the rotating dihedral angle is highlighted in light blue. The bcps are numbered like in the text and colored like in the graphs. The new hydrogen bond in the $\mathrm{H} 1$ structure is highlighted with a red dotted line. 


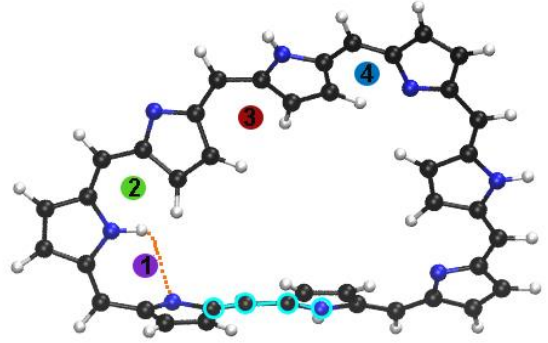

M2

Figure 11.Investigated bond critical points in the molecular switch, where the varying dihedral angle is highlighted in light blue. The bcps are numbered like in the text and colored like in the graphs. The new hydrogen bond is highlighted with a red dotted line.

The bond metallicity is highly correlated to the ELF value for these conformers $\left(R^{2}>0.99\right.$, Figure SI.10). Following from our analysis on the regular porphyrin, we have only investigated half of the critical points in the octaphyrin macrocycle, focusing on the side of the rotating dihedral angle. We use the same nomenclature as above, with the bond critical point next to the perturbation denoted as 1 and the index keeps increasing in a clock-wise manner. Thus, 2 and 8 are the closer bcps to the perturbation and so on.

The changes induced by the topological switch are extremely local in nature. The variation of the bond metallicity at bcps 2, 3, 4 are negligible with respect to that of bcp1 (Figure 12 and SI.11). The bond metallicity of $b c p 1$ increases dramatically when the dihedral angle reaches $60^{\circ}$ for $\mathrm{H} 1$ and $\mathrm{M} 2$ (Table SI.2). This increase is due to the formation of a hydrogen bond indicated in red in Figures 10 and 11. For the same non-covalent interaction framework (H1 and $\mathbf{M 2}$ ), Hückel leads to a better conductance, but the biggest structureconductance change is expected as the M1-H1 conformational change is induced. This is specially so if we take into account that bcp1 was initially the one that was related to the lowest metallicity, and hence, it could be thought of as the conductance limiting step.

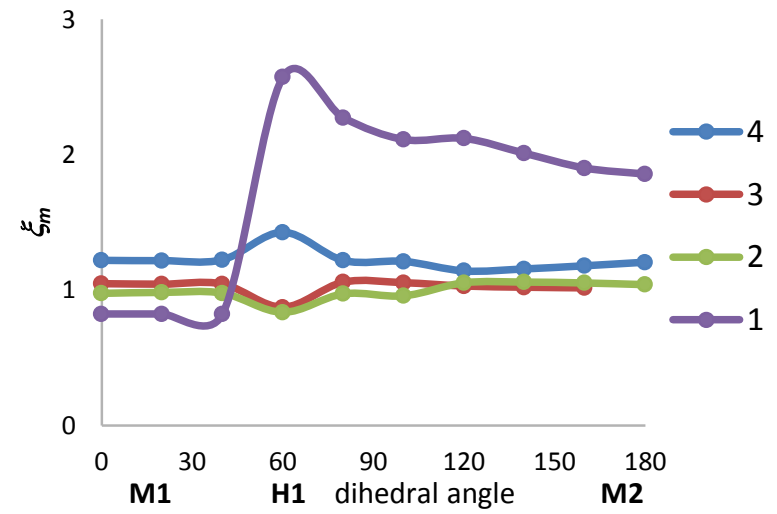

Figure 12. Evolution of the bond metallicity with the amplitude of the dihedral angle (molecules M1, H1, M2).

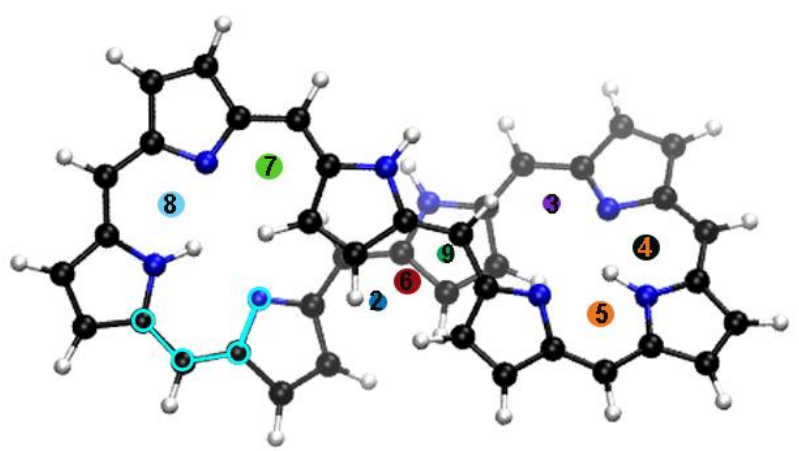

$\mathrm{H2}$

Figure 13. Hückel topology $\mathbf{H} \mathbf{2}$ with the bond critical points colored according to thei position the varying dihedral angle is highlighted in light blue.

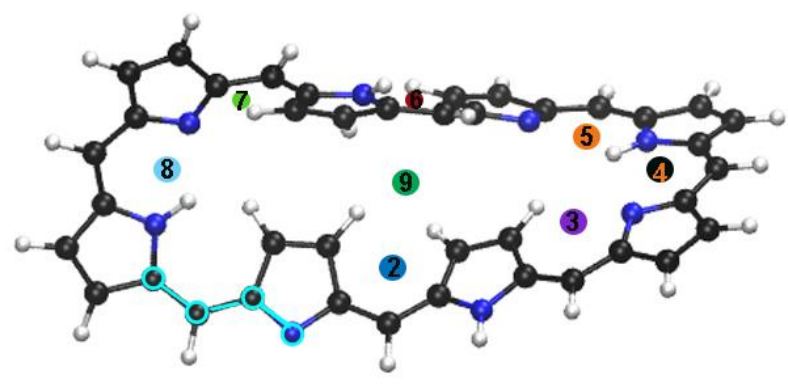

M3

Figure 14. Möbius topology M3 with the bond critical points colored according to their position and the rotating dihedral angle is highlighted in light blue.

As a second study case, we investigated a one-step Hückel-Möbius interconversion that displays a smooth potential energy surface (Figure SI.12). This Hückel-Möbius transition corresponds to the untwisting of the twisted-Hückel conformer H2 (Figure 13) that results in the Möbius conformer M3 (Figure 145). The bond critical point above the changing dihedral angle is denoted again as $b c p 1$ and the following bcps are named clockwise from this point on, so that the bcps close to the perturbation are again $b c p 2$ and $b c p 8$. The interesting thing about this transformation is that a $\pi-\pi$ staking interaction appears at the half-twist (H2), which is denoted as bcp9. The bcps close to this $\pi-\pi$ interaction are $b c p 6$ and $b c p 9$ in our graphs (Figure 13).

Although the electron density topological analysis carried out with our modified version of TOPMOD reveals 11 non-covalent bcps at the global minimum of the PES $\left(\mathbf{H} \mathbf{2}, d=0^{\circ}\right)$, we only evaluated measures of metallicity at the most important bcps ( 8 in total). In fact, according to the evolution of the laplacian during the interconversion, several non-covalent interactions gradually disappear as the amplitude of the dihedral angle increases. Since $b c p 1$ was soon undetectable by the topological analysis, its measures of metallicity were not used in the analysis of the macrocycle. 
The bond metallicity $\xi_{m}$ changes are depicted in Figure 15. They remain highly correlated to ELF values during the whole interconversion $\left(\mathrm{R}^{2}>0.93\right.$, (Table SI.3, Figure SI.13). The bond critical points lying in the vicinity of the perturbation $(b c p 8, b c p 2)$ are more sensitive to the variation of the dihedral

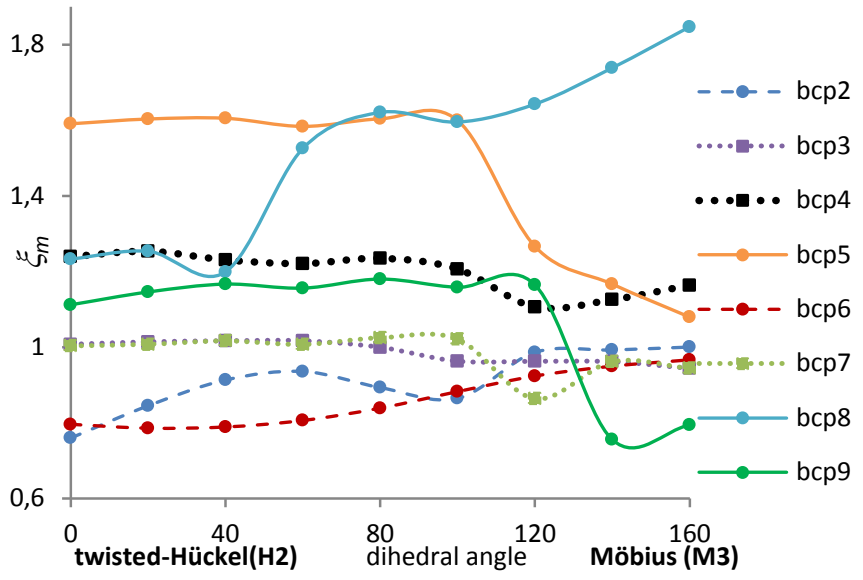

Figure 15. Evolution of the bond metallicity index at several critical points of the molecule with the amplitude of the dihedral angle (rotation from $\mathbf{H} \mathbf{2}$ to $\mathbf{M} 3$ ).

angle, highlighting the local nature of the metallicity in this system. However, since the Hückel-Möbius transition involves the creation of a $\pi-\pi$ staking interaction, the corresponding $b c p(b c p 9)$ and its neighbor (bcp6) also present a non-negligible variation. In fact, owing to the loss of a $\pi-\pi$ stacking interaction, the bond metallicity of $b c p 5$ and $b c p 9$ decreases for larger amplitudes of the dihedral angle.

Delocalization at the $b c p s$ close to the twist $(b c p 2, \mathrm{bcp} 6$ and $b c p 8)$ increases when going from the twisted-Hückel $\mathbf{H 2}$ to the Möbius topology M3. This behavior differs slightly from one index to another. In comparison to the bond metallicity, the delocalization index seems to be less position dependent. All in all, although the $b c p$ closest to the perturbation still presents the highest variation in the measures of metallicity, delocalization is not fully local since the important changes also occur associated to the folding.

Thus, we have seen that local measures of metallicity are able to cast the changes in delocalization upon conformational switch in octaphyrins. In those cases where the conformation remains open (so that there is no net change of interactions associated with the twist), the changes remain rather local, which can be used in the qualitative prediction of delocalization and conductivity in porphyrins and expanded porphyrins. In those cases where there is a fold, the change in interactions introduces a non local behavior which has to be taken into account.

\section{Conclusions}

We have reviewed several local indexes of metallicity based on the analysis of electron density, ELF and the kinetic energy density at the bond critical point. Since these quantities have usually been applied to solid state, we have first analysed its applicability to molecules. Firstly, we have seen that these measures are mutually coherent and that they can be used in very small molecular switches. Next, the relationship between the bond metallicity index and conductivity was probed. We have seen that the delocalization indicator is able to provide a qualitative picture of the electronic properties of $p$-phenylene and $p$-xylylene chains that is in agreement with the molecular conductance of both systems. Finally, we checked a larger system to test the applicability of local indexes to describe changes in a switch. We found that delocalization in model small switches is a local quantity because their variation is less pronounced far from the perturbation.

Based on these results, we have tested two topological switches based on the [36]octaphyrin. Our results demonstrated that the bond metallicity and delocalization index provide a qualitative description of the electron delocalization in octaphyrins, which are in agreement with ELF topological analysis. According to the measures of bond metallicity, we observed that the variation of the electron delocalization was local when the topological change was restricted to one part of the molecule and semi-local for global topological changes (e.g. folding). In the local cases, our approach enables to identify which conformational switch would be more efficient from an electronic device perspective. As our indices were able to grasp the difference between those two interconversions, they could also be used to perform a qualitative analysis in octaphyrins. These studies are expected to be applicable to other macrocycles and will be the object of future studies.

\section{Acknowledgements}

M. A. thanks the Fund for Scientific Research-Flanders FWO for a postdoctoral fellowship $(12 \mathrm{~F} 4416 \mathrm{~N})$ and the Free University of Brussels (VUB) for financial support. F. D. P wishes to acknowledge the VUB for a Strategic Research Program.

\section{References}

1 M. Jurow, A. Schuckman, J. Batteas, C. Drain, CoordChem Rev., 2010, 254, 2297-2310

2 B. L. Feringa, W. R. Brwone, Molecular Switches; WileyVCH: Weinheim, Germany, 2011; J. Andréasson, U. Pischel, Chem. Soc. Rev., 2010, 39, 174-188; B. Gui, X. Meng, Y. Chen, J. Tian, G. Liu, C. Shen, M. Zeller, D. Yuan, C. Wang, Chem. Mater., 2015, 27, 6426-6431; T. Woller, J. ContrerasGarcía, P. Geerlings, Frank De Proft, and M. Alonso, Understanding Molecular Switching Properties of Octaphyrins, submitted to PCCP.

3 M. Stępień, B. Szyszko and L. Latos-Grażyński, J. Am. Chem. Soc., 2010, 132 (9)

4 M. Stępień, N. Sprutta, L. Latos-Grażyński, Angew. Chem. Int. Ed. 2011, 50, 4288-4340

5 S. Saito, A. Osuka, Angew. Chem. Int. Ed. 2011, 50, 43424373 
6 M. Alonso, P. Geerlings, F. De Proft, Chem. Eur. J., 2013, 19, 1617-1628

7 M. Torrent-Sucarrat, J. M. Anglada, J. M. Luis, J. Chem. Phys. 2012, 137, 184306

8 G. Karthik, J. Min Lim, A. Srinivasan, C. H. Suresh, D. Kim, T.K. Chandrashekar, Chem. Eur. J., 2013,19, 17011-17020

9 D. Hrsak, M. A. Pertejo, Al M. Lamshabi, A. Muranaka, A. Ceulemans, Chem. Phys. Lett., 2013, 586,148-152

10 M. Alonso, P. Geerlings, F. De Proft, Chem. Eur. J. 2012, 18, 10916-10928

11 M. Alonso, P. Geerlings, F. De Proft, Phys. Chem. Chem. Phys. 2014, 16, 14396-14407

12 J. M. Lim, J-Y. Shin, Y. Tanaka, S. Saito, A. Osuka and D. Kim, J. Am. Chem. Soc., 2010, 132, 3105-3114.

13 J. Y. Shin, K. S. Kim, M. C. Yoon, J. M. Lim, Z. S. Yoon, A. Osuka, D. Kim, Chem. Soc. Rev. 2010, 39, 2751-2767.

14 W. Kohn, Phys. Rev. Lett., 1996,76, 3168-3171

15 T. Marino, M. C. Michelini, N. Russo, E. Sicilia, M. Toscano, Theor Chem Acc, 2012, 131-141.

16 X.P. Li, W. Nunes, D. Vanderbilt, Phys. Rev. B, 1993, 47 , 10891-10894.

17 R. Baer, M. Head-Gordon, Phys. Rev. Lett., 1997,79, 3962396

18 S. Jenkins, J. Phys. Condens. Matter, 2002,14,10251-10263

19 P. W. Ayers, S. Jenkins, Comp. Theor. Chem., 2014,1053,112-122

20 N. Seriani, ,J. Phys.-Condens. Matter,2010, 22, 255502.

21 R.F.W. Bader, Atoms in Molecules: A Quantum Theory, Clarendon, Oxford, 1990.

22 R.F.W. Bader, T.T. Nguyendang, Adv. Quantum Chem., 1981, $14,63-124$.

23 P.L.A. Popelier, Atoms in Molecules: An Introduction, Pearson, Harlow, 2000.

24 C.F. Matta, R.F.W. Bader, J Phys. Chem. A , 2006,110, 63656371.

25 R.F.W. Bader, J.Phys. Chem. A, 1998, 102, 7314-7323.

26 R.F.W. Bader, Y. Tal, S.G. Anderson, T.T. Nguyen-Dang, Isr. J. Chem., 1980, $19,8-29$.

27 P. Mori-Sanchez, A.M. Pendas, V. Luana, , J. Am. Chem. Soc., 2002,124,14721-14723

28 S. Jentkins, J. Phys. Condens. Matter., 2012, 14, 1025110263.

29 A. Savin, O. Jepsen, J. Flad, O. K. Andersen, H. Preuss, H. G. von Schnering, Angew. Chem. Int., 1992,31,187-187

30 A.D. Becke, K.E. Edgecombe, J. Chem. Phys., 1990, 92, 53975403.

31 J. Contreras-Garcia, J. M. Recio, Theor. Chem. Acc., 2011, 128,411

32 B. Silvi, C. Gatti, J. Phys. Chem. A., 2000, 104, 947-953.

33 S. Jenkins, S.R. Kirk, P.W. Ayers, W.F. Kuhs, R. Soc. Chem., 2006, 265-272.

34 V. Tsirelson, A. Stash, Chem. Phys. Lett., 2002, 351(1-2),142148.

35 H.L. Schmider, A.D. Becke, J. Chem. Phys. , 2002, 116, 31843193.

36 H.L. Schmider, A.D. Becke, Theochem.-J. Mol. Struct. , 2000, 527, 51-61

37 S. Jenkins, P. W. Ayers, S.R. Kirk, P. Mori-Sánchez, A. Martín Pendás, Chem. Phys. Lett., 2009, 1(471), 174-177.

38 S.K. Ghosh, M. Berkowitz, R.G. Parr, Proc. Natl. Acad. Sci., 1984, 81, 8028-8031.

39 L.H. Thomas, Proc. Camb. Philos. Soc., 1927, 23,542-548.

40 E. Fermi, Z. Phys.,1928, 48,73-79.

41 J.M. Tao, G. Vignale, I.V. Tokatly, Phys. Rev. Lett., 2008, 100 ,206405.

42 Gaussian 03, Revision C.02, M. J. Frisch, G. W. Trucks, H. B. Schlegel, G. E. Scuseria, M. A. Robb, J. R. Cheeseman, J. A. Montgomery, Jr., T. Vreven, K. N. Kudin, J. C. Burant, J. M.
Millam, S. S. Iyengar, J. Tomasi, V. Barone, B. Mennucci, M. Cossi, G. Scalmani, N. Rega, G. A. Petersson, H. Nakatsuji, M. Hada, M. Ehara, K. Toyota, R. Fukuda, J. Hasegawa, M. Ishida, T. Nakajima, Y. Honda, O. Kitao, H. Nakai, M. Klene, X. Li, J. E. Knox, H. P. Hratchian, J. B. Cross, V. Bakken, C. Adamo, J. Jaramillo, R. Gomperts, R. E. Stratmann, O. Yazyev, A. J. Austin, R. Cammi, C. Pomelli, J. W. Ochterski, P. Y. Ayala, K. Morokuma, G. A. Voth, P. Salvador, J. J. Dannenberg, V. G. Zakrzewski, S. Dapprich, A. D. Daniels, M. C. Strain, O. Farkas, D. K. Malick, A. D. Rabuck, K. Raghavachari, J. B. Foresman, J. V. Ortiz, Q. Cui, A. G. Baboul, S. Clifford, J. Cioslowski, B. B. Stefanov, G. Liu, A. Liashenko, P. Piskorz, I. Komaromi, R. L. Martin, D. J. Fox, T. Keith, M. A. Al-Laham, C. Y. Peng, A Nanayakkara, M. Challacombe, P. M. W. Gill, B. Johnson, W. rhen M W/ W/nno $C$ Fnnzalez, and J. A. Pople, Gaussian, Inc., Wallingford CT, 2004.

43 Gaussian 09, Revision D.01, M. J. Frisch, G. W. Trucks, H. B. Schlegel, G. E. Scuseria, M. A. Robb, J. R. Cheeseman, G. Scalmani, V. Barone, B. Mennucci, G. A. Petersson, H. Nakatsuji, M. Caricato, X.Li, H. P. Hratchian, A. F. Izmaylov, J. Bloino, G. Zheng, J. L. Sonnenberg, M. Hada, M. Ehara, K.Toyota, R. Fukuda, J. Hasegawa, M. Ishida, T. Nakajima, Y. Honda, O. Kitao, H. Nakai, T. Vreven, J.A. Montgomery, Jr., J. E. Peralta, F. Ogliaro, M. Bearpark, J. J. Heyd, E. Brothers, K. N. Kudin, V. N.Staroverov, R. Kobayashi, J. Normand, K. Raghavachari, A. Rendell, J. C. Burant, S. S. Iyengar, J.Tomasi, M. Cossi, N. Rega, J. M. Millam, M. Klene, J. E. Knox, J. B. Cross, V. Bakken, C. Adamo, J.Jaramillo, R. Gomperts, R. E. Stratmann, O. Yazyev, A. J. Austin, R. Cammi, C. Pomelli, J. W.Ochterski, R. L. Martin, K. Morokuma, V. G. Zakrzewski, G. A. Voth, P. Salvador, J. J. Dannenberg, S.Dapprich, A. D. Daniels, Ö. Farkas, J. B. Foresman, J. V. Ortiz, J. Cioslowski, and D. J. Fox, Gaussian,Inc., Wallingford CT, 2009

44 S. Noury, X. Krokidis, F. Fuster, B. Silvi, Comput. Chem. 1999, 23, 597-604.

45 M-C Yoon, S. Cho, M. Suzuki, A. Osuka, D. Kim, J. Am. Chem. Soc., 2009, 131(21), 7360-7367.

46 P. Schleyer, Chemical Reviews, 2001, 101, 5

47 I. Alkorta, I. Rozas, M. L. Jimeno, J. Elguero, Struct. Chem. ,2001, 12(6),459-464

48 J. R. Lane, J. Contreras-Garcia, J.-P. Piquemal, B. J. Miller, H. G. Kjaergaard, J. Chem. Theory Comp.,2013, 9, 3263

49 N. Ramos-Berdullas, M. Mandado, .Chem. Eur. J., 2013,19,3646-3654.

50 M. Alonso, P. Geerlings, F. De Proft, Chem. Eur. J., 2013, 19, 1617-1628. 